\title{
Intrinsic Fluid Interfaces and Nonlocality
}

\author{
Eva M. Fernández, ${ }^{1,2, *}$ Enrique Chacón, ${ }^{1,3}$ Pedro Tarazona, ${ }^{3,4}$ Andrew O. Parry, ${ }^{5,6}$ and Carlos Rascón 6 \\ ${ }^{1}$ Instituto de Ciencia de Materiales de Madrid, CSIC, 28049 Madrid, Spain \\ ${ }^{2}$ Departamento de Física Fundamental, Universidad Nacional de Educación Distancia, Madrid 28040, Spain \\ ${ }^{3}$ Instituto de Ciencia de Materiales Nicolás Cabrera, Universidad Autónoma de Madrid, Madrid 28049, Spain \\ ${ }^{4}$ Departamento de Física Teórica de la Materia Condensada, Condensed Matter Physics Center (IFIMAC), \\ Universidad Autónoma de Madrid, Madrid 28049, Spain \\ ${ }^{5}$ Department of Mathematics, Imperial College London, London SW7 2BZ, United Kingdom \\ ${ }^{6}$ GISC, Departamento de Matemáticas, Universidad Carlos III de Madrid, 28911 Leganés, Spain
}

(Received 18 June 2013; published 30 August 2013)

\begin{abstract}
We present results of an extensive molecular dynamics simulation of the structure and fluctuations of a liquid-gas interface, close to its triple point, in a system with cutoff Lennard-Jones interactions. The equilibrium density profile, averaged and (shape dependent) constrained intrinsic density profiles together with the fluctuations of the interfacial shape are extracted using an intrinsic sampling method. The correlation between fluctuations in the interfacial shape and in the intrinsic density show that the latter is not due to rigid translations of some underlying profile, as is most commonly assumed. Instead, over the whole range of wavelengths from the system size down to the molecular diameter, we see wave-vector dependent behavior in good agreement with a nonlocal interfacial Hamiltonian theory specifying the shape dependence of the intrinsic profiles.
\end{abstract}

DOI: 10.1103/PhysRevLett.111.096104

PACS numbers: 68.03.Hj, 05.70.Np, 68.03.Kn

A detailed understanding of the interface between coexisting fluid phases requires us to model physics at different length scales [1-8]. First, there is a microscopic range spanning the molecular size to the bulk correlation length, which determines the surface tension $\gamma$. This range is often associated with an intrinsic interfacial density profile $\varrho(z)$, which describes how the density changes across an instantaneous interfacial configuration. Second, there is a mesoscopic range from the bulk correlation length to the system size. At this scale, the interface resembles a taut drumskin described by a height variable $\ell(\mathbf{x})$, and one often considers the intrinsic density profile to be a sharp kink from liquid to gas [9]. The capillary wave fluctuations of the interface are characterized by a parallel correlation length and a roughness, which are much larger than the bulk correlation length, and indeed diverge in the absence of pinning fields (gravity, walls, or finite size). Almost all the controversies surrounding the physics of fluid interfaces arise directly from the uneasy marriage between these two descriptions. This marriage is often forced by supposing that the intrinsic profile $\varrho(z)$ rigidly shifts with the undulations of the interfacial fluctuations. Then, the equilibrium profile $\rho(z)$ is obtained by a simple convolution

$$
\rho(z)=\int d \ell \varrho(z-\ell) P(\ell)
$$

where $P(\ell)$ is the probability distribution function for the interfacial height, which is determined by an interfacial Hamiltonian $H[\ell]$ describing the energy cost of a given interfacial shape $\ell(\mathbf{x})$. In the standard capillary wave theory of a free interface, $P(\ell)$ is a Gaussian of square width
$\xi_{\perp}^{2}=\left(k_{B} T / 2 \pi \gamma\right) \log (\Lambda L)$, where $L$ is the system size and $\Lambda$ is a microscopic cutoff [9]. Thus, capillary wave fluctuations dramatically broaden the interface and, indeed, in the absence of pinning fields, wash out any structure in the equilibrium $\rho(z)$ [10].

In this Letter, we show that this concept of a rigidly shifted intrinsic interfacial profile is inconsistent with two very different recent developments in the theory of interfacial phenomena. Each of these have set out to precise the physics associated with the microscopic intrinsic interface and the mesoscopic interfacial Hamiltonian descriptions: The first one, called the intrinsic sampling method (ISM), gives a precise definition of the interfacial shape $\ell(\mathbf{x})$ for any given molecular configuration, and allows one to obtain an intrinsic profile $\varrho(z)$ computed by molecular dynamics [11-14]. The second is the derivation of a nonlocal interfacial Hamiltonian from a continuum microscopic model in the manner of van der Waals, which accurately models the energy of long wavelength interfacial configurations and, in particular, the interaction with nearby walls $[15,16]$. The nonlocal nature of this Hamiltonian has proved to be essential to overcome several long-standing problems associated with the order of wetting transitions in the Ising model [17-19]. Using the ISM, we show here that the correlation between the intrinsic profile and the interfacial position obtained in a simulation of a molecular fluid with Lennard-Jones interactions matches precisely the predictions of the nonlocal theory, and is different to the standard capillary wave assumption of a rigidly shifted profile.

Consider the interface between bulk liquid and gas localized near the $z=0$ plane by a suitable external field. 
In classical statistical mechanics, the equilibrium density profile $\rho(z)$ is obtained via the ensemble average $\rho(z)=$ $\langle\hat{\rho}\rangle$ of the density operator [3]

$$
\hat{\rho}(\mathbf{x}, z)=\sum_{i}^{N} \delta\left(\mathbf{x}-\mathbf{x}_{i}\right) \delta\left(z-z_{i}\right),
$$

where we have split the usual positional delta function $\delta\left(\mathbf{r}-\mathbf{r}_{i}\right)$ into contributions perpendicular and parallel to the interface. Thus, $\left\{\mathbf{r}_{i}=\left(\mathbf{x}_{i}, z_{i}\right)\right\}$ denote the positions of our $N$ particles, and $\langle\cdots\rangle$ is the ensemble average over all molecular configurations, which are weighted by the Boltzmann factor $\exp \left(-\mathcal{H} / k_{B} T\right)$. Here, $\mathcal{H}$ is the microscopic Hamiltonian, specified by the intermolecular forces and external field.

For systems with short-ranged forces, density functional theory (DFT) predicts that $\rho(z)$ decays into the liquid bulk as $\rho(z)-\rho_{\text {liq }}=a e^{-\alpha z}+b e^{-\beta z}+\cdots$, where we have assumed that liquid occupies the region $z>0$. The same decays but with different amplitudes describe the radial distribution function $(g(r)-1) r=a^{\prime} e^{-\alpha r}+b^{\prime} e^{-\beta r}+\cdots$. One usually finds a real exponent $\alpha=\kappa$ and a complex pair $\beta=\kappa_{\mathrm{osc}} \pm i q_{\mathrm{osc}}$, representing the molecular layering [20]. For $\rho(z)$ near a wall and $g(r)$, one typically finds $b$, $b^{\prime} \gg a, a^{\prime}$, so that the oscillatory effects dominate. However, $a \gg b$ for the liquid-vapor interface, even at DFT level, which ignores capillary waves larger than 10 molecular diameters [21]. This means that the density profile shows a smooth decay over these distances, irrespective of being above or below the Fisher-Widom line, that separates regions with smooth or oscillatory asymptotic decay [22]. The inclusion of capillary waves serves to emphasize the importance of the smoothly decaying contribution, since they amplify the coefficient $a \rightarrow a e^{\kappa^{2} \xi_{\perp}^{2} / 2}$, but suppress the oscillatory coefficient $b \rightarrow b e^{-\left(q_{\mathrm{osc}}^{2}-\kappa_{\mathrm{osc}}^{2}\right) \xi_{\perp}^{2} / 2}$.

Our first challenge is to determine the position of the interface $\ell(\mathbf{x})$ at any point $\mathbf{x}=(x, y)$ parallel to the interface from our particle positions $\mathbf{r}_{i}=\left(\mathbf{x}_{i}, z_{i}\right)$. Various approaches are available [11,23-29], a critical revision of which has been reported in two recent reviews [30,31]. For example, one may use a local Gibbs dividing surface, which simply balances the number of particles within the bulk phases either side of the interface. This is computationally easy but does not distinguish interfacial modes from the bulklike compressibility fluctuations of the liquid near the surface, and produces an interface that corrugates too much at shorter wavelengths [12]. Here, we use the ISM, which defines $\ell(\mathbf{x})$ using a many-particle percolative analysis of the molecular configurations [11-14]. Crucial to this is the joint identification of the particles belonging to the "outermost layer" of the liquid, and the definition of $\ell(\mathbf{x})$ as the surface of minimal area through that set of particles $[13,14]$. Although computationally more demanding, this remains sound down to wavelengths of 2 or 3 molecular diameters and, thereby, successfully separates interfacial and bulk fluctuations. In particular, it has been used in recent studies of wetting [32,33].

Having identified the interface shape from any molecular configuration, we can now define the intrinsic density profile

$$
\varrho(z)=\langle\hat{\rho}(\mathbf{x}, z+\ell(\mathbf{x}))\rangle,
$$

where the "intrinsicness" arises because the particle coordinates $z_{i}$ are measured with respect to the interface location. Clearly, this function depends on the recipe used to determine the interface location from the molecular positions. For example, the lazy choice of an interface location $\ell(\mathbf{x})=0$ independent of the molecular configurations leads to an intrinsic profile identical to the equilibrium one, which does not eliminate any capillary wave broadening. An appropriate choice, on the other hand, would lead to a function $\varrho(z)$ independent of the system size and, hence, the influence of capillary waves.

To understand the connection between equilibrium and intrinsic structure, we need to perform the ensemble average $\langle\cdots\rangle$ in two stages, which will allows us to define a third density profile. Rather than average over all molecular configurations, we first only consider those which lead to the same interfacial shape $\ell(\mathbf{x})$. We denote this average $\langle\cdots\rangle_{\ell}$. When this average is applied to the density operator, we obtain a constrained profile

$$
\rho_{\ell}(\mathbf{x}, z)=\langle\hat{\rho}(\mathbf{x}, z)\rangle_{\ell},
$$

which is a functional of the interface shape $\ell(\mathbf{x})$ and a function of $\mathbf{x}$ and $z$. If we now further average over all distinct interfacial shapes, translational invariance parallel to the interface is restored, and we reobtain $\rho(z)$ :

$$
\rho(z)=\left\langle\rho_{\ell}(\mathbf{x}, z)\right\rangle_{\text {all } \ell},
$$

which is the exact version of the heuristic expression (1). Similarly, we can also use $\rho_{\ell}(\mathbf{x}, z)$ to obtain the intrinsic profile by shifting the $z$ coordinate:

$$
\varrho(z)=\left\langle\rho_{\ell}(\mathbf{x}, z+\ell(\mathbf{x}))\right\rangle_{\text {all } \ell} .
$$

These final two formulas involve the average $\langle\cdots\rangle_{\text {all }} \ell$ which can also be written in terms of a functional integral over all interfacial shapes, each with a Boltzmann factor $\exp \left(-H[\ell] / k_{B} T\right)$. Thus, we may rewrite (5) as

$$
\rho(z)=\frac{1}{Z} \int D \ell \rho_{\ell}(\mathbf{x}, z) e^{-H[\ell] / k_{B} T} .
$$

Here, $H[\ell]$ is the mesoscopic interfacial Hamiltonian and $Z$ is the partition function. Note that, in general, the exact evaluation of (7) requires knowledge far beyond the one-point distribution $P(\ell)$ and, therefore, $\rho(z)$ cannot be expressed as the convolution (1) without additional assumptions.

It is clear from Eq. (7) that the constrained density $\rho_{\ell}(\mathbf{x}, z)$ is key to the connection between the equilibrium and intrinsic structure, since it contains information about 
the interfacial shape. Unfortunately, a direct measurement of this function, even in a molecular simulation, is prohibitively difficult. However, the information about the shape dependence can be obtained from analysis of the two-point correlation function between the constrained density at position $\mathbf{r}=(\mathbf{x}, z)$ and the interfacial height at $\mathbf{x}^{\prime}$ :

$$
C(x, z)=\left\langle\rho_{\ell}(\mathbf{x}, z) \ell\left(\mathbf{x}^{\prime}\right)\right\rangle_{\text {all } \ell}
$$

where $x=\left|\mathbf{x}-\mathbf{x}^{\prime}\right|$. To see this, we recall that in standard descriptions of intrinsic profiles, it is assumed that the constrained interfacial profile is a local rigid shift of the intrinsic profile

$$
\rho_{\ell}(\mathbf{x}, z) \approx \varrho(z-\ell(\mathbf{x}))
$$

With this assumption, Eq. (7) reduces identically to the standard convolution of Eq. (1). In fact, Eq. (9) is explicit in the derivation of an interfacial Hamiltonian $H[\ell]$ in the important work of Fisher and Jin, in the context of wetting theory $[34,35]$. These authors start, not from a molecular description but rather, from a coarse-grained continuum Landau-Ginzburg-Wilson (LGW) model, and the assumption (9) leads directly to a local interfacial Hamiltonian. For a free interface, this has the standard form $H[\ell]=$ $\int d \mathbf{x} \gamma(\nabla \ell)^{2} / 2$, where $\gamma$ is the surface tension.

However, recent developments have improved the derivation of the interfacial Hamiltonian by explicitly allowing for the shape dependence of $\rho_{\ell}(\mathbf{x}, z)$ [16]. For example, within the continuum LGW description, the constrained intrinsic density decays as

$$
\rho_{\ell}(\mathbf{x}, z) \sim \int d s^{\prime} K\left(\sqrt{\left|\mathbf{x}^{\prime}-\mathbf{x}\right|^{2}+\left(\ell\left(\mathbf{x}^{\prime}\right)-z\right)^{2}}\right),
$$

where we have substracted the bulk liquid density. Here, $d s^{\prime}=\sqrt{1+\left(\nabla \ell\left(\mathbf{x}^{\prime}\right)\right)^{2}} d \mathbf{x}^{\prime}$ is the local interfacial area element, and $K(r)=\kappa \exp (-\kappa r) / 2 \pi r$ is essentially the bulk correlation function of the LGW model. In this nonlocal description, the constrained density depends on the full interfacial shape $\ell$, in contrast to the local description (9) for which the decay is simply $\rho_{\ell}(\mathbf{x}, z) \approx e^{-\kappa(z-\ell(\mathbf{x}))}$. This local assumption only emerges in the long wavelength limit of (10). Not surprisingly, allowing for the full shape dependence of $\varrho_{\ell}$ leads to an effective Hamiltonian $H[\ell]$ containing many-body interfacial interactions. Note that, with the nonlocal constrained density (10), the expression (5) does not reduce to the simple convolution (1), implying that the intrinsic density and equilibrium profile are not related directly.

Importantly, the local and nonlocal theories predict different behavior for the decay of the correlation function $C(x, z)$, which is most easily seen in its 2D Fourier transform $\tilde{C}(q, z)$ w.r.t. $\mathbf{x}$. According to the nonlocal description, this exhibits a wave-vector dependent decay length,

$$
\tilde{C}(q, z) \sim e^{-\sqrt{\kappa^{2}+q^{2}} z},
$$

in contrast to the local assumption (9), which predicts $\tilde{C}(q, z) \sim e^{-\kappa z}$. Of course, at $q=0$ these theories are identical but, for any nonzero $q$, they are very different for sufficiently large values of $z$.

To illustrate this, we present in Fig. 1 the results of a toy calculation which compares the local and nonlocal profiles $\rho_{\ell}(\mathbf{x}, z)$ for a pure harmonic interfacial shape $\ell(\mathbf{x})=$ $\hat{\ell}_{q} \cos (q x)$ with $\hat{\ell}_{q}=0.1 \sigma$ and $q \sigma=2$. Here, the local density is a rigid shift of the free interface profile, and the nonlocal one is obtained from a simple LGW calculation (see Supplemental Material [36]). It is clear from the contour plots that the densities are increasingly different away from the interface. While this does not visibly manifest itself in the $\mathbf{x}$ averaged profile $\rho_{\mathbf{x}}(z)$ (which mimics a thermal average), there is a very clear distinction in the decays of $\tilde{C}(q, z)$.

To test the validity of these ideas, we have performed large scale molecular dynamics (MD) $N V T$ simulations of a Lennard-Jones (LJ) fluid, using the software package LAMMPS [37]. With direct numerical access to molecular configurations and the interfacial shape $\ell(\mathbf{x})$ defined via the ISM, we have computed $\rho(z), \varrho(z)$, and $\tilde{C}(q, z)$. The LJ potential is truncated at $r_{c}=4.4 \sigma$, where $\sigma$ is the standard LJ length scale. This choice of cutoff, larger than the usual
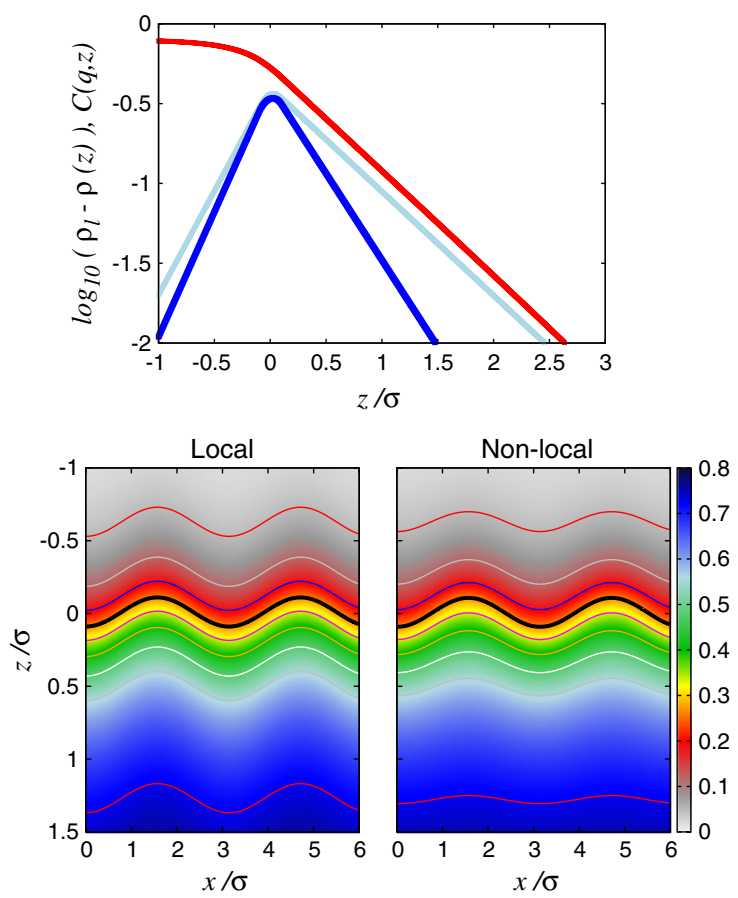

FIG. 1 (color online). Bottom: Density distributions $\rho_{\ell}(x, z)$ obtained with the local (right) and nonlocal (left) assumptions for a toy harmonic corrugation, $\ell(x)=\hat{\ell}_{q} \cos (q x)$. The thin lines are contour lines, the black one being the location of the interface $z=\ell(x)$. Top: The local and nonlocal $\mathbf{x}$-averaged profiles $\rho_{\mathbf{x}}(z)$ (red line) are indistinguishable on this scale. However, the local (light blue) and nonlocal (blue) $\tilde{C}(q \sigma=2.0, z)$ are markedly different. 


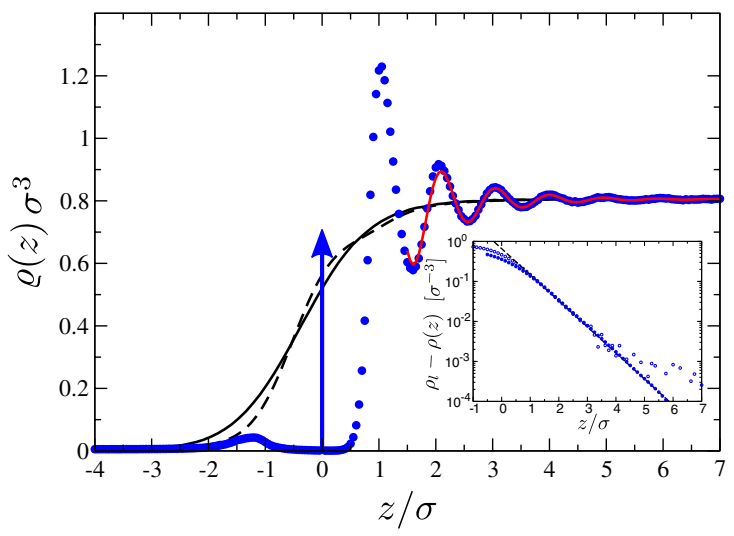

FIG. 2 (color online). Plots of the equilibrium density profile $\sigma^{3} \rho(z)$ (full line) and intrinsic density profile $\varrho(z) \sigma^{3}$ (blue circles) vs $z / \sigma$, determined by the ISM. The arrow represents a delta function contribution corresponding to the outmost layer of the intrinsic interface. The tail of $\varrho(z) \sigma^{3}$ is well fitted by $y=$ $0.805-0.4 \exp (-1.52 x)+0.92 \exp (-1.03 x) \cos [6.58(x-1.13)]$ (red line), showing a monotonically decaying term with the same $\kappa \sigma \approx 1.50 \pm 0.05$ as in $\rho(z)$ (see inset), and a slower decaying oscillatory term with inverse length scale $\kappa_{\mathrm{osc}} \sigma \approx 1.03$. Also shown (dashed line) is the prediction of the standard capillary wave theory based on Eq. (1). In the inset, the empty circles show the raw data while the full circles are the results after applying a Gaussian filter with width $\Delta=0.5 \sigma$, which extends the range of $z$ over which the exponential decay may be observed (See Supplemental Material [36]).

$2.5 \sigma$, reduces the vapor density and improves the accuracy of the ISM. For the same reason, simulations are performed close to the triple point temperature, corresponding to $k_{B} T / \epsilon=0.763$, where $\epsilon$ is the $\mathrm{LJ}$ interaction strength. Our system consists of 176441 particles forming a liquid slab surrounded by vapor in a box of size $L_{x}=L_{y}=41.83 \sigma$ and $L_{z}=250 \sigma$ with periodic boundary conditions. Very large simulation times were necessary to ensure a good average of the thermal fluctuations for small $q$. For this reason, systems were initially equilibrated for $2 \times 10^{6} \mathrm{MD}$ steps. 5000 measurements were taken every $5000 \mathrm{MD}$ steps, with time steps of $d t=5 \times 10^{-3} \sigma \sqrt{m / \epsilon}$.

Figure 2 shows the equilibrium profile $\rho(z)$ and intrinsic profile $\varrho(z)$ obtained from the MD simulations. Over the range of $z$ considered, the equilibrium profile is monotonic and decays exponentially into the liquid with inverse decay length $\kappa \sigma=1.5 \pm 0.05$ (see inset). In contrast, the intrinsic profile shows oscillations arising from packing effects, and its decay is well fitted by a mix of a pure exponential decay, as in the mean density, and an oscillatory decaying term: $\varrho(z) \approx \rho_{\text {liq }}+a e^{-\kappa z}+b e^{-\kappa_{\mathrm{osc}} z} \cos \left(q_{\mathrm{osc}} z+\theta\right)$. Here, the value of $\kappa$ is the same as obtained from $\rho(z)$, while $\kappa_{\text {osc }} \sigma \approx 1.03 \pm 0.01$ is the same inverse length describing the decay of oscillations in the radial distribution function $g(r)$. The fact that $\kappa_{\mathrm{osc}}<\kappa$ indicates that we are below the Fisher-Widom line. Indeed, $g(r)$ is itself very well fitted by $(g(r)-1) r \approx b^{\prime} e^{-\kappa_{\text {osc }} r} \cos \left(q_{\text {osc }} r+\theta\right)$. As discussed earlier,

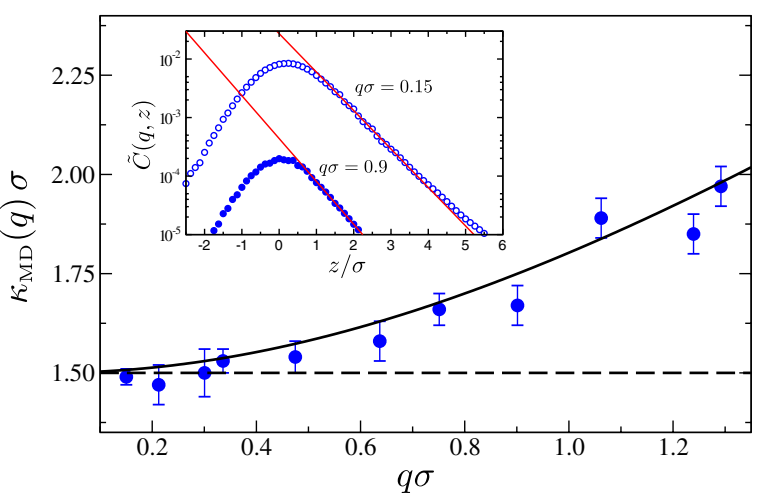

FIG. 3 (color online). Simulation results for $\kappa(q)$ (blue circles) together with the predictions of the nonlocal [continuous line, $\kappa(q)=\sqrt{\kappa^{2}+q^{2}}$ ] and local [dashed line, $\kappa(q)=\kappa$ ] interfacial Hamiltonian theories. The inset shows two log plots of the dimensionless correlation function $\tilde{C}(q, z)$ as a function of $z / \sigma$ for wave numbers $\sigma q=0.15$ (empty circles) and $\sigma q=0.9$ (filled circles).

the equilibrium profile must also show oscillations at very large distances, but their amplitude is near negligibly small due to the capillary wave broadening. Also shown is the prediction of the standard capillary wave theory based on Eq. (1) using the microscopic cutoff determined by the ISM. As can be seen, the convolution washes out the oscillatory structure of $\varrho(z)$ but does not lead to an entirely satisfactory description of the equilibrium profile. Figure 3 and the inset illustrate the main numerical results of our Letter concerning the properties of $\tilde{C}(q, z)$. The main figure shows the measured values of $\kappa_{\mathrm{MD}}(q)$ as a function of the wave number, obtained from the fit $\tilde{C}(q, z) \propto e^{-\kappa_{\mathrm{MD}}(q) z}$. The inset shows two examples of this fit for $q \sigma=0.15$ and $q \sigma=0.9$. For $q \sigma<0.4$ the local and nonlocal predictions for $\kappa_{\mathrm{MD}}(q)$ cannot be distinguished, and both recover the same exponential decay extracted from the equilibrium density. However, for larger values of $q$, the measured $\kappa_{\mathrm{MD}}(q)$ is in excellent agreement with the prediction of the nonlocal theory $\kappa(q)=\sqrt{\kappa^{2}+q^{2}}$ (continuous line), and differs markedly from the prediction of the local theory $\kappa(q)=\kappa$ (dashed line).

In summary, the central result of our Letter is the agreement between the MD simulation results and the nonlocal interfacial Hamiltonian theory for the decay of $\tilde{C}(q, z)$ over the whole range of wavelengths from system size to molecular size. This demonstrates that the constrained profile $\rho_{\ell}(\mathbf{x}, z)$ is shape dependent, beyond what is allowed for in the approximation (9) of standard capillary wave theory. The robust agreement strongly suggests that the important shape dependence of $\rho_{\ell}(\mathbf{x}, z)$ is determined by the smoothly decaying exponential contribution, which is correctly described by Eq. (10), rather than by oscillatory terms arising from molecular layering, which are entirely absent in the LGW model that underlies the NL theory. 
A. O.P. acknowledges the support of the bank of Santander and UCIIIM for a Chair of Excellence. E. M. F., E. C., P. T., and C. R. acknowledge support from grants MODELICO (Comunidad de Madrid) and FIS201022047-C05 (Ministerio de Educación y Ciencia).

*emfernandez@fisfun.uned.es

[1] M. Wertheim, J. Chem. Phys. 65, 2377 (1976).

[2] J. Weeks, J. Chem. Phys. 67, 3106 (1977).

[3] R. Evans, Adv. Phys. 28, 143 (1979).

[4] J.S. Rowlinson and B. Widom, Molecular Theory of Capillarity (Claredon Press, Oxford, 1982).

[5] J. Percus, in Fluid Interfacial Phenomena, edited by C. Croxton (John Wiley, New York, 1986), p. 1.

[6] K. R. Mecke and S. Dietrich, Phys. Rev. E 59, 6766 (1999).

[7] D. Aarts, M. Schmidt, and H. Lekkerkerker, Science 304, 847 (2004).

[8] E. M. Blokhuis, J. Chem. Phys. 130, 014706 (2009).

[9] F. P. Buff, R. A. Lovett, and F. H. Stillinger, Phys. Rev. Lett. 15, 621 (1965).

[10] J. Weeks, W. van Saarloos, D. Bedeaux, and E. Blokhuis, J. Chem. Phys. 91, 6494 (1989).

[11] E. Chacón and P. Tarazona, Phys. Rev. Lett. 91, 166103 (2003).

[12] P. Tarazona and E. Chacón, Phys. Rev. B 70, 235407 (2004).

[13] E. Chacón and P. Tarazona, J. Phys. Condens. Matter 17, S3493 (2005).

[14] E. Chacón, E. M. Fernández, D. Duque, R. DelgadoBuscalioni, and P. Tarazona, Phys. Rev. B 80, 195403 (2009).

[15] A. O. Parry, J.M. Romero-Enrique, and A. Lazarides, Phys. Rev. Lett. 93, 086104 (2004).

[16] A. O. Parry, C. Rascón, N.R. Bernardino, and J. M. Romero-Enrique, J. Phys. Condens. Matter 18, 6433 (2006).

[17] A. O. Parry, C. Rascón, N.R. Bernardino, and J.M. Romero-Enrique, Phys. Rev. Lett. 100, 136105 (2008).
[18] A. O. Parry and C. Rascón, J. Low Temp. Phys. 157, 149 (2009).

[19] L. Pang, D. P. Landau, and K. Binder, Phys. Rev. Lett. 106, 236102 (2011).

[20] P. Tarazona, E. Chacón, and E. Velasco, Mol. Phys. 101, 1595 (2003).

[21] R. Checa, E. Chacón, and P. Tarazona, Phys. Rev. E 70, 061601 (2004).

[22] R. Evans, J. R. Henderson, D. C. Hoyle, D. A. Parry, and Z. A. Sabeur, Mol. Phys. 80, 755 (1993).

[23] F. H. Stillinger, J. Chem. Phys. 76, 1087 (1982).

[24] S. Pandit, D. Bostick, and M. Berkowitz, J. Chem. Phys. 119, 2199 (2003).

[25] J. Chowdhary and B. M. Ladanyi, J. Phys. Chem. B 110, 15442 (2006).

[26] D. Zhukhovitskii, J. Chem. Phys. 125, 234701 (2006).

[27] L. Partay, G. Hantal, P. Jedlovszky, A. Vincze, and G. Horvai, J. Comput. Chem. 29, 945 (2008).

[28] M. Jorge and M. Cordeiro, J. Phys. Chem. C 111, 17612 (2007).

[29] A. Willard and D. Chandler, J. Phys. Chem. B 114, 1954 (2010).

[30] M. Jorge, P. Jedlovszky, and N. Cordeiro, J. Phys. Chem. C 114, 11169 (2010).

[31] M. Jorge, G. Hantal, P. Jedlovszky, and N. Cordeiro, J. Phys. Chem. C 114, 18656 (2010).

[32] E. M. Fernández, E. Chacón, and P. Tarazona, Phys. Rev. B 84, 205435 (2011).

[33] E. M. Fernández, E. Chacón, and P. Tarazona, Phys. Rev. B 86, 085401 (2012).

[34] M.E. Fisher and A. J. Jin, Phys. Rev. Lett. 69, 792 (1992).

[35] A. J. Jin and M.E. Fisher, Phys. Rev. B 48, 2642 (1993).

[36] See Supplemental Material at http://link.aps.org/ supplemental/10.1103/PhysRevLett.111.096104 for a more detailed explanation about how the density plotted in Fig. 1 is calculated, and for description of the Gaussian filter used in order to reduce the noise and to improve the accuracy of our simulations results.

[37] S. J. Plimpton, J. Comput. Phys. 117, 1 (1995). 